\title{
Dynamic shift of the epibacterial communities on commercially cultivated Saccharina japonica from mature sporophytes to sporelings and juvenile sporophytes
}

\author{
Qi Han ${ }^{1} \cdot$ Xiaoyang Zhang ${ }^{1} \cdot$ Lirong Chang $^{2} \cdot$ Luyang Xiao $^{2} \cdot$ Rafiq Ahmad $^{1} \cdot$ Mahasweta Saha $^{3} \cdot$ Hongyan Wu $^{4}$. \\ Gaoge Wang ${ }^{1}$ (D)
}

Received: 3 July 2020 / Revised and accepted: 5 November 2020

(C) Springer Nature B.V. 2021

\begin{abstract}
The supply of healthy sporelings plays a crucial role in the successful aquaculture of commercially cultivated brown kelp Saccharina japonica. Epibacteria are of critical importance for maintaining seaweed health and development. Thus, this study aimed to investigate the epibacterial community associated with mature sporophytes and 1.5- and 2.0-month-old sporelings, as well as juvenile sporophytes of $S$. japonica by using $16 \mathrm{~S}$ rRNA gene amplicon sequencing and predicted the bacterial functional profiles. We found that Proteobacteria, Verrucomicrobia, Bacteroidetes, and Planctomycetes were the main phyla, while Loktanella and Rubritalea and Persicirhabdus were the dominant genera for all four developmental stages. Highly abundant core microbiota included Persicirhabdus, Loktanella, Litorimonas, and Rubritalea. Further analysis of the predicted functions showed that different metabolic functions were involved at different developmental stages. More metabolic functions were found in 2-month-old sporelings and mature sporophytes. Both the diversity, composition of the epiphytic bacterial communities, and metabolic functions changed according to different developmental stages at least in 1.5- and 2-month-old sporelings. Our results not only provide fundamental knowledge for the epibacteria associated with $S$. japonica but will also help nursery farms in preventing and mitigating the disease outbreaks by monitoring the variations of the epibacterial communities at the nursery stage.
\end{abstract}

Keywords Epibacterial communities $\cdot$ 16S rRNA gene $\cdot$ Phaeophyceae $\cdot$ Saccharina japonica $\cdot$ Sporelings

\section{Introduction}

It is well-known that the surface of macroalgae harbors a large number of microbes which have been shown to be important to macroalgal development, defense, metabolic activity, etc. (Egan et al. 2013). During the last decade, the diversity and dynamics of the epibacterial communities associated with

Hongyan $\mathrm{Wu}$

sdwuhongyan@126.com

Gaoge Wang

gaogewang@hotmail.com

1 College of Marine Life Science and Institute of Evolution \& Marine Biodiversity, Ocean University of China, Qingdao 266003, China

2 Weihai Changqing Ocean Science \& Technology Co., Ltd, Rongcheng 264316, China

3 Marine Ecology and Biodiversity, Plymouth Marine Laboratory, Prospect Place, Plymouth PL1 3DH, UK

4 Ludong University, Yantai 264025, China macroalgae have been intensively investigated due to advances in high-throughput DNA sequencing technology. One of the concordant results is that the diversity and the epiphytic microbial cell density appear to be highly affected by the seasonal growth or life cycle (Lemay et al. 2018). In general, the number of epibacteria is highest at the tip, i.e., old tissues, and lowest on the basal meristem, i.e., new tissues of Laminaria spp. (Laycock 1974; Mazure and Field 1980), of Saccharina latissima (Staufenberger et al. 2008), of Cystoseira compressa (Mancuso et al. 2016), of Macrocystis pyrifera (Michelou et al. 2013), and eight other sympatric species (Lemay et al. 2018). This indicates that algal thallus age might affect microbial community development.

Saccharina japonica is one of the most important commercially cultivated seaweeds in China. Since the summersporeling nursery technology was invented in 1958, it is possible to supply a large-scale quantity of healthy sporelings for the cultivation of S. japonica (Tseng 1994). Usually, the summer-sporeling nursery starts in early August and ends in mid-October in greenhouses. The nursery stage includes 
collecting the mature sporophytes with sporangia, stimulating the sporangia to release the zoospores and monitoring the development from zoospores to sporelings. Like land crops, S. japonica suffers from different diseases at the nursery stage (Xiang 2001; Wang et al. 2014). The kelp microbiome can be an indicator of overall host health (Marzinelli et al. 2015). So far, it is well-known that some of the epibacteria of S. japonica can become pathogenic whenever the temperature and light intensity increase (Wang et al. 2008, 2014). Compared to wild macroalgae, knowledge related to the diversity and the dynamics of the epibacterial communities on both cultivated healthy and diseased sporelings and sporophytes of $S$. japonica are scarce. Recently, Zhang et al. (2020) found that Proteobacteria and Bacteroidetes were the main phyla and Halomonas was the most dominant bacterial genus of both healthy and diseased sporophytes of cultivated S. japonica during the harvest season (from April to June 2015). Epibacterial communities were affected by the sampling time and sampling site. However, there are no reports yet on the epibacterial community associated with the sporelings of $S$. japonica at the nursery stage. Therefore, understanding how microbial communities are structured on the sporelings of $S$. japonica can provide insights into the mechanisms by which microbial associations are assembled and maintained on healthy sporelings of S. japonica. Furthermore, it can also help to identify the pathogenic bacteria for the disease occurrence at the nursery stage.

In this study we characterized the dynamic shifts of the diversity of epibacterial communities associated with mature sporophytes, sporelings, and juvenile sporophytes of commercially cultivated $S$. japonica using 16 S rRNA gene amplicon sequencing. Additionally, we also analyzed the function profiles based on the 16S rRNA gene database. Our results not only enrich the fundamental knowledge of epibacteria associated with the sporelings of $S$. japonica but also can help nursery farms in monitoring the variation of epibacteria to prevent or predict the disease outbreaks.

\section{Materials and methods}

\section{Samples of epibacteria of S. japonica}

Epibacterial samples at four different developmental stages of Saccharina japonica (Fig. 1) were collected at the nursery farm at Qingyutan, Rongcheng, Shandong Province, China ( $37^{\circ} 16^{\prime}$ $03.07^{\prime \prime} \mathrm{N}, 122^{\circ} 56^{\prime} 64.84^{\prime \prime}$ E). Mature sporophytes (MS) with sporangia were cultivated in the sea and were collected just before the release of zoospores on 10 August 2018. Within $4 \mathrm{~h}$, samples were transported to the laboratory in polyethylene bags and kept cool using ice packs. Epibacteria were sampled by swabbing $50 \mathrm{~cm}^{2}$ of the surface of sporangia with a sterile cotton swab near the middle section of each mature sporophyte.
It usually takes 1.5 months for the zoospores to develop into 13-mm sporelings which could cover the surface of the substratum curtains. In order to collect the epibacteria of sporelings but not the bacteria attached to surface substratum curtains, we had to choose 1.5-month-old sporelings for sampling epibacteria (Fig. 1). During the nursery stage, 1.5-month-old sporelings (S1, 1-3 mm in length) and 2-month-old sporelings (S2, 10 $20 \mathrm{~mm}$ in length) grew on the substratum curtains $(40 \times$ $115 \mathrm{~cm}$ ) in the greenhouses. Epibacteria of 1.5-month-old and 2-month-old sporelings were collected from the sporelings attached to three random substratum curtains as three replicates by swabbing with the sterile cotton swabs on 26 September and on 11 October, respectively. Each substratum curtain was divided into 8 equal sections. One cotton swab was used for collecting epibacteria from each of 8 sections of each substratum curtain. Finally, 8 total cotton swabs were pooled into a $50-\mathrm{mL}$ sterile tube and treated as one replicate of the substratum curtains. Epiphytic bacterial samples were stored in dry ice during the transport to the laboratory. Those sporelings which were transferred into the sea and cultivated for 1 month were assigned as juvenile sporophytes (JS, $30-40 \mathrm{~cm}$ in length). Epibacterial samples of juvenile sporophytes were collected on 22 November 2018. The sampling method of epibacteria of juvenile sporophytes was the same as those of mature sporophytes. Three epibacterial replicates were sampled at each developmental stage, respectively, and were kept frozen at $20{ }^{\circ} \mathrm{C}$ until DNA extraction.

\section{Analysis of epibacterial communities}

Microbial DNA was extracted using the HiPure Soil DNA Kits (Magen, Guangzhou, China) according to the manufacturer's protocols. The $16 \mathrm{~S}$ rDNA V3-V4 region of the ribosomal RNA gene was amplified by PCR $\left(95^{\circ} \mathrm{C}\right.$ for $2 \mathrm{~min}$, followed by 27 cycles at $98{ }^{\circ} \mathrm{C}$ for $10 \mathrm{~s}, 62^{\circ} \mathrm{C}$ for $30 \mathrm{~s}$, and $68^{\circ} \mathrm{C}$ for $30 \mathrm{~s}$, and a final extension at $68^{\circ} \mathrm{C}$ for $10 \mathrm{~min}$ ) using primers 341F: 5'-CCTACGGGNGGCWGCAG-3'; 806R:5'GGACTACHVGGGTATCTAAT-3', where the barcode is an eight-base sequence unique to each sample. PCR reactions were performed in triplicate with $50 \mu \mathrm{L}$ mixture containing $5 \mu \mathrm{L}$ of $10 \times$ KOD buffer, $5 \mu \mathrm{L}$ of $2.5 \mathrm{mM}$ dNTPs, $1.5 \mu \mathrm{L}$ of each primer $(5 \mu \mathrm{M}), 1 \mu \mathrm{L}$ of KOD polymerase, and $100 \mathrm{ng}$ of template DNA. Amplicons were extracted from $2 \%$ agarose gel and purified according to the instructions using AxyPrep DNA Gel Extraction Kit (Axygen Biosciences, USA) and quantified by ABI SteponePlus real-time PCR system (Life Technologies, USA). The purified amplicon was pooled in equal moles and sequenced $(2 \times 250)$ on the Illumina platform according to the standard protocol.

The low-quality or ambiguous reading segments were removed to obtain high-quality tags data. The resulting sequence was compared with the database (Gold database) through the UCIME algorithm (Edgar et al. 2011) to detect 
Fig. 1 Developmental stages of mature sporophytes, 1.5 - and 2.0month-old sporelings and juvenile sporophytes of $S$. japonica. a Mature sporophytes $(\mathrm{MS}, \mathrm{bar}=30 \mathrm{~cm})$ with sporangia, the inset showed the sporangia (bar $=3 \mathrm{~cm}$ ); $\mathbf{b}$ the substratum curtain $($ bar $=10 \mathrm{~cm})$ with 1.5-month-old sporelings (S1), the inset exhibited the 1-3$\mathrm{mm}$ sporelings $(\mathrm{bar}=1 \mathrm{~mm})$; $\mathbf{c}$ the substratum curtain (bar = $10 \mathrm{~cm}$ ) with 2-month-old sporelings (S2), inset showed the 10-20-mm sporelings (bar = $2 \mathrm{~mm}$ ); d juvenile sporophytes (JS, bar $=3 \mathrm{~cm})$. The size of the substratum curtains was $40 \times$ $115 \mathrm{~cm}$
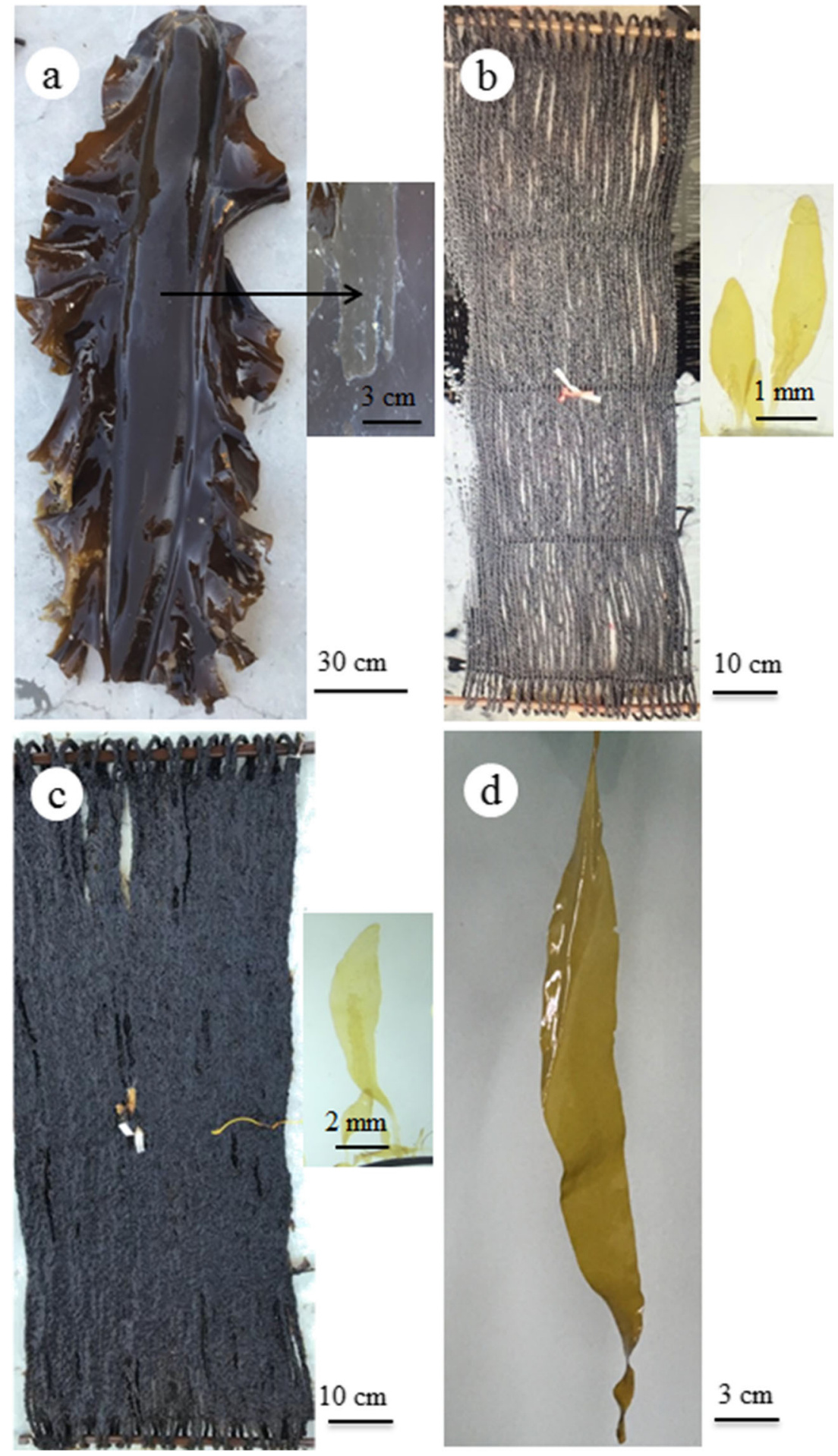

the chimeric sequence, and finally the chimeric sequence was removed to obtain the final high-quality data. The high-quality tags were clustered into operational taxonomic units (OTUs) of $\geq 97 \%$ similarity using the UPARSE pipeline (Edgar 2013). The sequences with the highest abundance were selected as the representative sequence for each cluster. The representative sequences were classified into organisms by a naive
Bayesian model using RDP classifier (Version 2.2) (Wang et al. 2007) based on SILVA Database (Version 128) (Pruesse et al. 2007) (https://www.arb-silva.de/), with the confidence threshold values ranging from 0.8 to 1 . The sequences were classified from phylum to genus level. On the basis of this classification, sequences of chloroplast and mitochondria origin were identified and subsequently 
removed from the dataset. The raw reads were deposited into the NCBI Sequence Read Archive (SRA) database (Accession Number SRP227413).

\section{Statistical analyses}

Chao1, Simpson diversity index, and Pielou's evenness were calculated in QIIME. Alpha index comparison between groups was calculated by Welch's $t$ test. Alpha index comparison among groups was computed by Tukey's HSD test. PCoA (principal coordinates analysis) of Bray-Curtis distances was generated in the $\mathrm{R}$ project Vegan package (Version 2.5.3) (Oksanen et al. 2010) and plotted in R project ggplot2 package (version 2.2.1) (Wichham 2016). The permutational analysis of variance (PERMANOVA) test was calculated in the R project Vegan package (version 2.5.3) (Oksanen et al. 2010). Clustsig package (Version 1.1) was used for the SIMPFOR analysis to determine significant differences by CLUSTER analysis. Venn diagram was drawn using the VennDiagram (v1.6.17) package (Chen and Boutros 2011) of the R project. Shared OUTs between four developmental stages were obtained by Wenn analysis. The core bacterial community was determined by the top 8 OUTs with higher relative abundance.

\section{Functional prediction of epiphytic bacterial communities}

KEGG pathway annotation of the OTUs was predicted by Tax4Fun (version 1.0, Aßhauer et al. 2015). Microbiome phenotypes were classified using BugBase (Ward et al. 2017). FAPROTAX database (Functional Annotation of Prokaryotic Taxa) and associated software were used for generating the ecological functional profiles (Louca et al. 2016).

\section{Results}

A total of 1,498,464 paired-end raw reads were obtained. After quality filtering and removal of chloroplast sequences, a total of $1,439,086$ sequences were obtained for subsequent analysis. All sequences were classified into 2314 OTUs ( $97 \%$ sequence similarity). These sequences were classified into 33 phyla, 78 classes, 117 orders, and 209 families. The rarefaction curve showed that species richness is high and evenly distributed (Fig. S1). A total of 33 bacterial phyla were detected in all samples. Among them, the main phyla were Proteobacteria, Verrucomicrobia, Bacteroidetes, and Planctomycetes (Fig. 2). According to the Kruskal-Wallis test (Table S1), 1.5-month-old sporelings hosted a significantly higher average abundance of Verrucomicrobia (72\%), while Proteobacteria was the most abundant in the mature sporophytes, 2-month-old sporelings, and juvenile sporophytes, with the average accounting for
$55 \%, 51 \%$, and $57 \%$, respectively. The rest of the other main bacterial phyla were Bacteroidetes and Planctomycetes. At the genus level, the dominant bacteria at each stage were more distinct. Loktanella (family Rhodobacteraceae) accounted for $20 \%$ of mature sporophytes. Persicirhabdus (family Verrucomicrobiaceae) was the dominant bacterium in 1.5month-old sporelings and juvenile sporophytes accounting for $16 \%$ and $28 \%$, respectively. Rubritalea (family Rubritaleaceae) was higher in 2-month-old sporelings, accounting for $17 \%$.

Regarding alpha diversity, the bacterial richness (the observed species) was significantly different between mature sporophytes and 2-month-old sporelings and between mature sporophytes and juvenile sporophytes (Fig. 3a; Table S2, $p<0.01$ ). Both the Shannon index (Fig. 3b) and Pielou's evenness (Fig. 3c) were not significantly different between mature sporophytes and sporelings/ juvenile sporophytes. Although the bacterial richness (the observed species) did not show a significant difference between 1.5- and 2.0-month-old sporelings (Fig. 3a), both the Shannon index (Fig. 3b) and Pielou's evenness (Fig. 3c) indicated that the bacterial population was significantly richer in 2-month-old sporelings (Table S3, $p<0.01$; Table S4, $p<0.01$ ).

The structure of epibacteria was significantly different at four developmental stages (PERMANOVA: $F_{2,17}=20.0436$, $p=0.001$, Table S5). The PCoA showed that the first axis explained the major part of the variation $(39.20 \%)$ and highlighted the large differences between 1.5-/2.0-month-old sporelings and juvenile/mature sporophytes, while the second axis explained the $26.88 \%$ of the total variation and reflected differences between 1.5- and 2.0-month-old sporelings (Fig. 4a). It can be seen that the bacterial community structure of different sample types was significantly different. According to the results of CLUSTER analysis (Fig. 4b), the samples at different developmental stages were divided into four groups at $80 \%$ similarity level, and there were significant differences between each two groups $(p<0.05)$.

A total of 172 OTUs were shared among the four developmental stages (Fig. 5, Table S6). The most conspicuous orders were Verrucomicrobiales and Caulobacterales. OTUs belonging to these groups were identified as core bacteria, including 119 Proteobacteria, 19 Bacteroidetes, 18 Planctomycetes, 13 Verrucomicrobia, 2 Actinomycetes, and 1 unrecognized phylum. The most abundant of these core bacteria are members of Verrucomicrobiales (OTU5), Verrucomicrobiales (Verrucomicrobiaceae, Persicirhabdus, OTU3), Rhodobacterales (Rhodobacteraceae, Loktanella, OTU6), Caulobacterales (Hyphomonadaceae, Litorimonas OTU9), Caulobacterales (Hyphomonadaceae, OTU10), Verrucomicrobiales (Verrucomicrobiaceae, Persicirhabdus, OTU11), and Verrucomicrobiales (Rubritaleaceae, Rubritalea, OTU12). The relative abundance of each core OTU fluctuated at each developmental stage. 

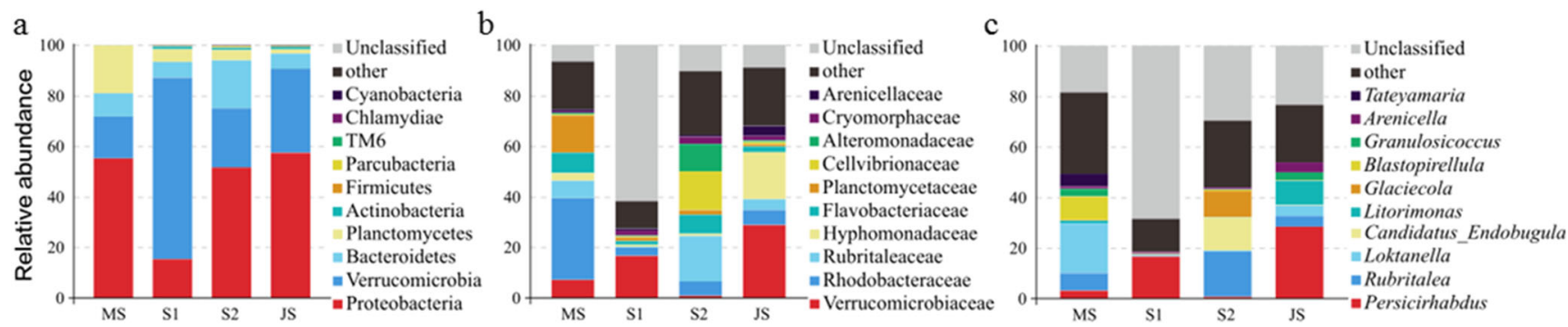

Fig. 2 Epiphytic bacterial community composition on S. japonica at four developmental stages at phylum (a), family (b), and genus (c) levels. MS, mature sporophytes with sporangia. S1, 1.5-month-old sporelings; S2, 2-month-old sporelings; JS, juvenile sporophytes

The epibacterial community function at each developmental stage was predicted by using Tax4Fun (Fig. 6) (Aßhauer et al. 2015). KEEG pathway differed in their abundance across the four developmental stages (Table S7). Glycine, serine, threonine metabolism, porphyrin and chlorophyll, amino sugar and nucleotide sugar, fructose and mannose, and bacterial secretion system were more abundant in epibacteria of mature sporophytes. Function related to aminoacyl-tRNA biosynthesis was more enriched in epibacteria of 1.5-month-old sporelings. Two-component system, pyruvate, arginine and proline metabolism, starch, and sucrose metabolism increased in epibacteria of 2-month-old sporelings. Flagellum synthesis of epibacteria was higher in abundance at the developmental stage of juvenile sporophytes.

\section{Discussion}

The microbiome associated with algae plays a key role in growth, development, and disease resistance (Egan et al. 2013; Wang et al. 2014; Saha and Weinberger 2019). In this study, we investigated the diversity and functional potential of epibacterial communities of commercially cultivated $S$. japonica at four developmental stages, i.e., mature sporophytes with sporangia, 1.5- and 2.0-month-old sporelings, and the juvenile sporophytes. We found that Proteobacteria, Verrucomicrobia, Bacteroidetes, and Planctomycetes were the dominant phyla for four developmental stages (Fig. 2). These taxonomic results at the phyla level are consistent with our recent results found in both healthy and diseased S. japonica over the harvest season (Zhang et al. 2020) and other macroalgal species, including Saccharina latissima (previously Laminaria saccharina) (Staufenberger et al. 2008), Laminaria hyperborea (Bengtsson et al. 2010), Ulva australis (Burke et al. 2011a), and Cystoseira compressa (Mancuso et al. 2016). However, in contrast to the dominant bacterial genus Halomonas spp. on the commercially cultivated S. japonica over the harvest season (Zhang et al. 2020), Loktanella (family Rhodobacteraceae), Persicirhabdus (family Verrucomicrobiaceae), and Rubritalea (family Rubritaleaceae) were the dominant genera for the mature sporophytes, 1.5-month-old sporelings/juvenile sporophytes, and 2-month-old sporelings, respectively. This indicated that different genera dominated at different developmental stages. Loktanella is the most populated genus in the Roseobacter group (Roseobacteraceae) and encompasses a total of 13 species, isolated from diverse marine samples (seawater, sediments, and beach sand) around the world. They are known to be involved in sulfur and carbon biogeochemical cycling and live in symbiosis with aquatic micro- and macro-

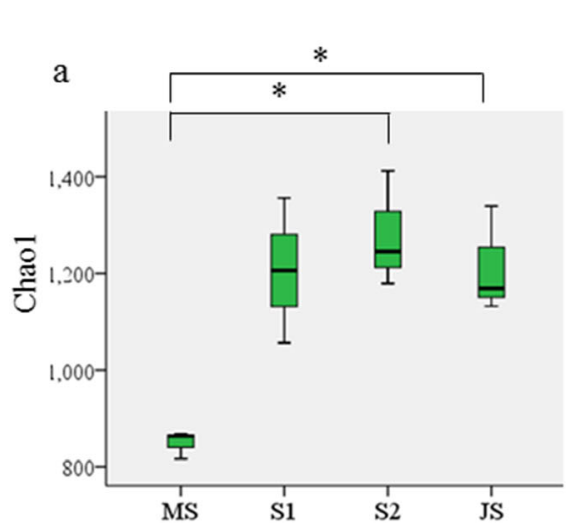

Fig. 3 Alpha diversity of the epibacterial community associated with $S$. japonica at four developmental stages. a Chaol species richness. b Shannon diversity index. c Pielou's evenness. Values are means \pm standard error $(n=3)$. Boxplots show extreme and lower whisker, lower
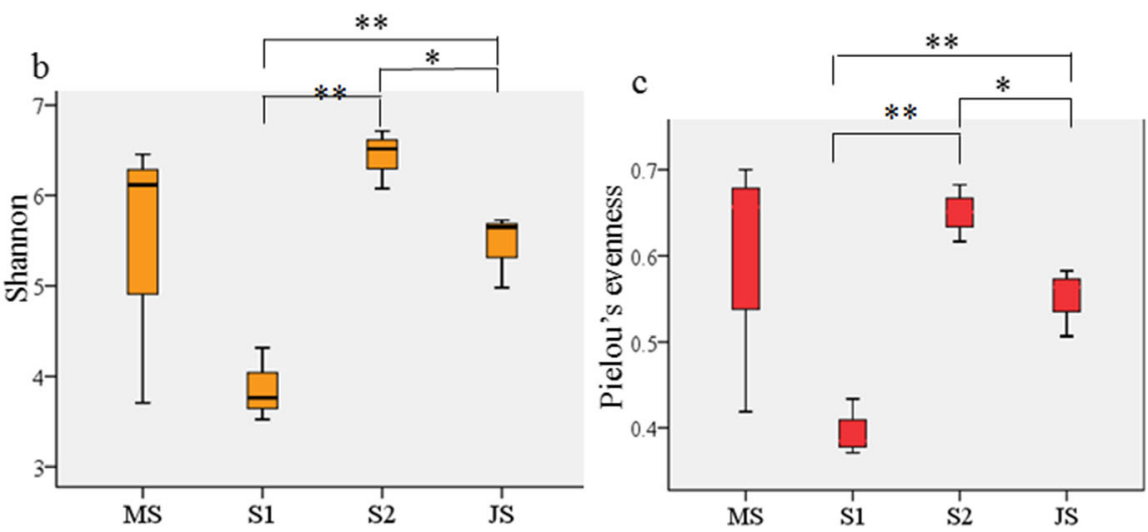

and upper quartiles (box), and median. MS, mature sporophytes with sporangia; S1, 1.5-month-old sporelings; S2, 2-month-old sporelings; JS, juvenile sporophytes. $* p<0.05 ; * * p<0.01$ 


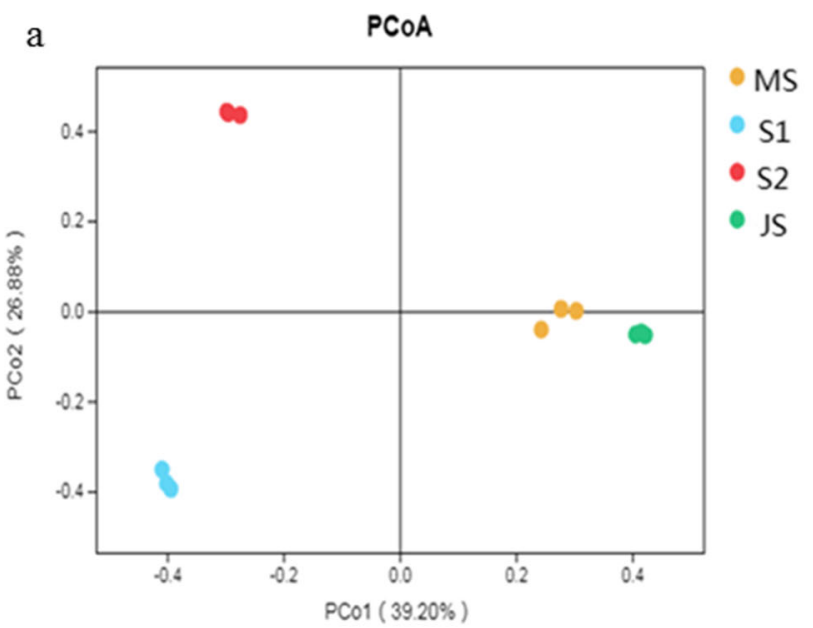

Fig. 4 The structure of the epibacterial community associated with $S$. japonica at four developmental stages. a Principal coordinate analysis (PCoA) plot of epibacterial communities of $S$. japonica based on the Bray-Curtis distance. Colors represent mature sporophytes (yellow, MS), 1.5-month-old sporelings (blue, S1), 2-month-old sporelings

organisms (Tsubouchiet al. 2013; Pujalte et al. 2014). Persicirhabdus has been isolated from a variety of marine environments including seawater (Yoon et al. 2007a), sediment (Yoon et al. 2008), and marine animals (Yoon et al. $2007 \mathrm{~b}$ ), indicating their presence in many marine environments. They have been demonstrated to be important for the biogeochemical cycle of carbon in the ocean (Yoon et al. 2008). With respect to Rubritalea belonging to the phylum Verrucomicrobia, only four of the five species were isolated from marine invertebrates and marine sediment (Song et al. 2018). These bacteria have the potential to affect the health and ecology of the hosts. Due to relatively less information available regarding the functions of Loktanella, Persicirhabdus, and Rubritalea, it is still unknown what kind of roles these dominant bacteria play during the development of $S$. japonica.

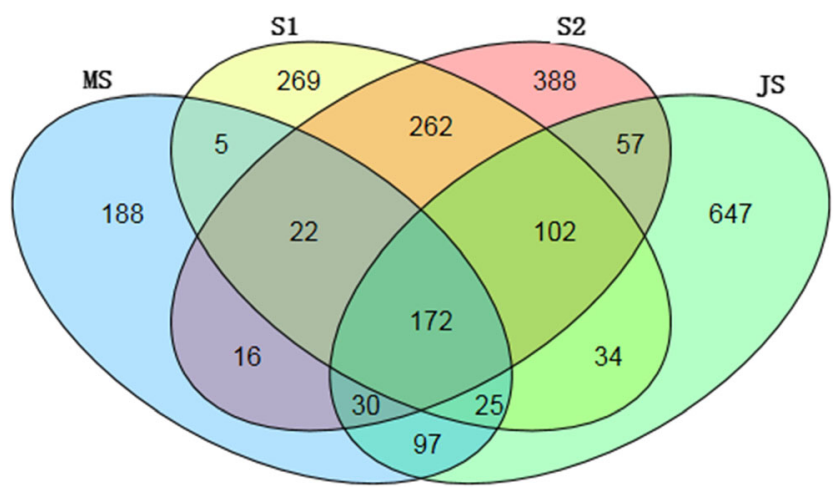

Fig. 5 Venn diagram showing the distribution of OTUs displays the extent of shared and exclusive OTUs between four developmental stages of $S$. japonica. MS, mature sporophytes with sporangia; S1, 1.5month-old sporelings; S2, 2-month-old sporelings; JS, juvenile sporophytes

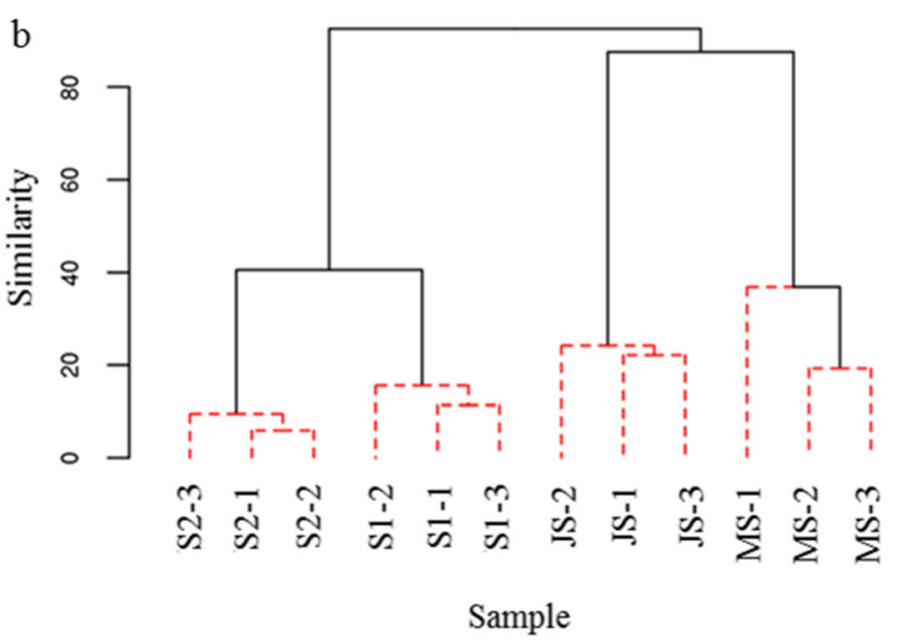

(red, S2), and juvenile sporophytes (green, JS). b Classification of epibacterial communities based on the CLUSTER analysis. Samples connected by solid black lines have significant differences (SIMPROF, $p<0.05$ ), while samples connected by red dashed lines have no significant difference (SIMPROF, $p>0.05$ )

In this study, 1.5-month-old sporelings $(1-3 \mathrm{~mm})$ and 2month-old sporelings $(10-20 \mathrm{~mm}$ ) were grown in the nursery greenhouses, whereas juvenile sporophytes and mature sporophytes with sporangia were cultivated in the sea. The epibacterial communities were characterized by lower OTU richness, evenness, and Shannon diversity indices on 1.5month-old sporelings when compared to the older 2-monthold sporelings (Fig. 3). Similar results were also reported in other macroalgae. Lower bacterial richness and species diversity have been found in newly grown kelp blades or meristematic region than older thallus tissues during the life cycle of L. saccharina (Staufenberger et al. 2008), with the tissue age of L. hyperborea (Bengtsson et al. 2012), over the annual growth cycle of Cystoseira compressa (Mancuso et al. 2016), in the growth season of Nereocystis luetkeana (Weigel and Pfister 2019), and during the harvest season of S. japonica (Zhang et al. 2020). This difference in the bacterial richness and species diversity has been attributed to the different photosynthesis activities and to the different exudates released by the alga at different life cycle stages. Our results together with previous studies indicate that the dynamic shifts of the epibacterial communities associated with both wild and commercially cultivated macroalgae change along with changes in the life cycle of the alga. However, Shannon indices and evenness of the epibacterial communities of juvenile and mature sporophytes (both cultivated in the sea) did not shift due to the life cycle pattern. Lower bacterial richness was found on mature sporophytes but there was no significant difference in both Shannon indices (Fig. 3b) and bacterial evenness (Fig. 3c). There are two possible reasons which may have resulted in this pattern. One reason is that the epibacterial samples of mature sporophytes came from the surface of sporangia. We speculate that the bacterial 
Fig. 6 Functional prediction of epibacterial community associated with S. japonica during four developmental stages. The KEGG pathway annotation of the OTUs was inferred using Tax4Fun (version 1.0, Aßhauer et al. 2015), respectively. MS, mature sporophytes with sporangia; S1, 1.5-month-old sporelings; S2, 2-month-old sporelings; JS, juvenile sporophytes

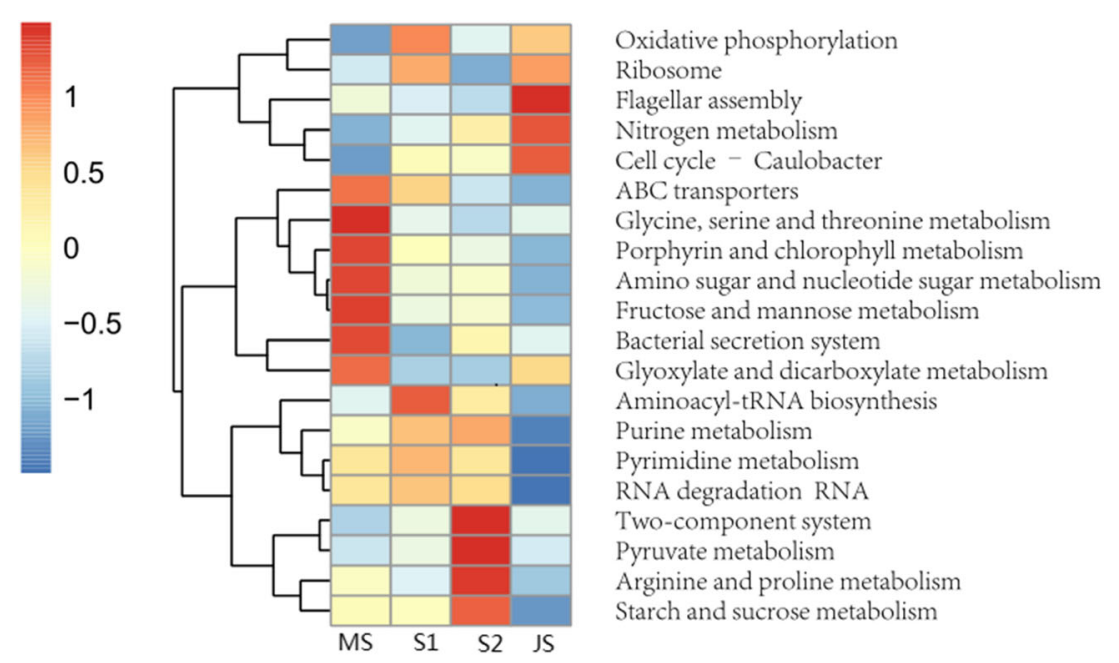

communities associated with the sporangia, which are related to the reproduction, may be different from the non-sporangia area of the mature sporophytes. So far, there is less knowledge on the epibacteria related to the sporangia, zoospores, and the very tiny sporelings in both wild and commercially cultivated seaweeds. Further studies are required to identify how the microbiome associated with seaweeds can vary due to the alteration of generations.

In total, 172 shared OTUs indicated the core bacteria shared across the four developmental stages (Table S6). These OTUs mainly belong to Proteobacteria, Bacteroidetes, Planctomycetes, Verrucomicrobia, and Actinomycetes. The most two conspicuous orders were Verrucomicrobiales (30.28\%) and Caulobacterales (11.94\%). At the genus level, the most abundant core bacteria were Persicirhabdus (OTU3), Loktanella (OTU6), Litorimonas (OTU9), and Rubritalea (OTU12), which were constantly fluctuating (in \% composition) across the four developmental stages. It has been reported that Gammaproteobacteria, Alphaproteobacteria, Firmicutes, and Actinobaceria were defined as the core/ shared communities in macroalgal-associated bacteria, while Pseudomonadales (class Gammaproteobacteria) might be more generalist symbionts found associated with most algal taxa (Hollants et al. 2013; Aires et al. 2015). However, some non-generalist core-associated bacteria were also found in seaweeds, for example, the core community of Burkholderiales in the green seaweed Caulerpa (Aires et al. 2015) and Sphingobacteriales in the microbiome of the red seaweed Asparagopsis (Aires et al. 2016). The different core community of Verrucomicrobiales and Caulobacterales as found in this study was in agreement with the hypothesis that these core communities might be tightly associated with the development of S. japonica.

Metabolic functions associated with the epibacteria of mature sporophytes, 1.5-month-old and 2.0-month-old sporelings, and juvenile sporophytes were predicted using
Tax4Fun. Our results indicated that the younger the sporelings or sporophytes were, the lesser metabolic functions were involved. For both sporelings (growing in greenhouses) and juvenile and mature sporophytes (cultivated in the sea), metabolic functions were found to be altered due to the change with different developmental stages. Compared to the 2month-old sporelings, only aminoacyl-tRNA biosynthesis was enhanced for 1.5-month-old sporelings, which indicated that protein biosynthesis was important at this stage. Twocomponent system, pyruvate metabolism, arginine and proline metabolism, starch, and sucrose metabolism increased in 2month-old sporelings when compared to 1.5-month-old sporelings. In the field cultivation, flagellum synthesis was accelerated in epibacterial communities from juvenile sporophytes, which might be important for bacteria to move towards the algal host surface during colonization and biofilm formation (Hossain and Tsuyumu 2006; Houry et al. 2010). However, more enriched bacterial functional metabolisms, including the metabolism of the amino acid, sugar, and bacterial secretion system, were found to be more abundant at the developmental stage of mature sporophytes. Common sugar metabolism probably enables bacteria to use the sugars as a source of carbon and energy and gain a competitive advantage in colonizing the host surface (Burke et al. 2011a). The metabolic functions involved may be related to the reproduction and the development of the sporangia. Metabolic functions of the epibacterial communities were investigated by comparing with metagenomes of other seaweeds. Burke et al. (2011b) found that the different epibacterial taxa of Ulva australis provided the core functions to the community and could form a functional guild. Recently, Aires et al. (2015) reported that a nitrate-reducing betaproteobacterium associated with Caulerpa sp. may increase their performances in the eutrophical/polluted sediments. Furthermore, they found that the high metabolic functions are associated with stress response and resistance to toxic compounds in the bacterial 
communities of Acidimicrobiales and Sphingomonadales in the invasive marine alga Asparagopsis taxiformis (Aires et al. 2016). These results implied that the bacterial communities and functional metabolisms are linked with each other and metabolic functions may be more useful in testing the ecological roles of bacterial communities (Burke et al. 2011b).

It is interesting that Persicirhabdus (family Verrucomicrobiaceae) was the dominant bacterium on both 1.5month-old sporelings and juvenile sporophytes accounting for $16 \%$ and $28 \%$, respectively. However, the metabolic functions are completely different between these two developmental stages. As there is little information about the metabolic functions of Persicirhabdus, we cannot discuss this in more detail. However, our results indicated that the same taxonomic group may play different functions at different developmental stages.

\section{Conclusions}

Saccharina japonica is one of the importantly commercially cultivated seaweeds worldwide. The supply of healthy sporelings plays a crucial role in successful cultivation of $S$. japonica. However, disease outbreaks during the artificial summer-sporeling nursery stage and field cultivation can cause a $20-25 \%$ production loss. This highlights the need for research into the ecological implications of altered epibacterial community structure during the period of the nursery stage. This study is the first step towards this goal, providing fundamental knowledge of $S$. japonica-associated epibacterial communities and paves the way for future studies during the nursery stage. Our data suggest that the diversity and the metabolic functions of the epibacterial communities get altered during the development at least for the sporeling stage. Thus, it is necessary to identify the characteristics of the assemblages of epibacterial communities and their metabolic/ gene functions, including the seawater during the whole life cycle. Complete knowledge of epibacterial communities associated with the sporelings at the nursery stage can help the nursery farms in preventing or mitigating the disease outbreaks.

Supplementary Information The online version contains supplementary material available at https://doi.org/10.1007/s10811-020-02329-4.

Acknowledgments This study was sponsored by the Marine S \& T Fund of Shandong Province for Pilot National Laboratory for Marine Science and Technology (Qingdao) (2018SDKJ0406-5); the National Natural Science Foundation of China (42076106; 41576158); the Fundamental Research Funds for the Central Universities (201822025); National Key R \& D Program of China (2018YFD0900305) and Rongcheng Marine Industrial Technology Research Institute, Ludong University (KF20180002).

\section{Compliance with ethical standards}

Conflict of interest The authors declare that they have no conflict of interest.Supplementary Information The online version contains supplementary material available at https://doi.org/10.1007/s10811-02002329-4.

\section{References}

Aires T, Moalic Y, Serrao EA, Arnaud-Haond S (2015) Hologenome theory supported by cooccurrence networks of species-specific bacterial communities in siphonous algae (Caulerpa). FEMS Microbiol Ecol 91:fiv067

Aires T, Serrao EA, Engelen AH (2016) Host and environmental specificity in bacterial communities associated to two highly invasive marine species (genus Asparagopsis). Front Microbiol 7:559

Aßhauer KP, Bernd W, Rolf D, Peter M (2015) Tax4Fun: predicting functional profiles from metagenomic $16 \mathrm{~S}$ rRNA data. Bioinformatics 31:2882-2884

Bengtsson MM, Sjøtun K, Øvreås L (2010) Seasonal dynamics of bacterial biofilms on the kelp Laminaria hyperborea. Aquat Microb Ecol 60:71-83

Bengtsson MM, Sjotun K, Lanzen A, Ovreas L (2012) Bacterial diversity in relation to secondary production and succession on surfaces of the kelp Laminaria hyperborea. ISME J 6:2188-2198

Burke C, Steinberg P, Rusch D, Kjelleberg S, Thomas T (2011a) Bacterial community assembly based on functional genes rather than species. Proc Natl Acad Sci U S A 108:14288-14293

Burke C, Thomas T, Lewis M, Steinberg P, Kjelleberg S (2011b) Composition, uniqueness and variability of the epiphytic bacterial community of the green alga Ulva australis. ISME J 5:590-600

Chen H, Boutros PC (2011) VennDiagram: a package for the generation of highly-customizable Venn and Euler diagrams in R. BMC Bioinformat 12:35

Edgar RC (2013) UPARSE: highly accurate OTU sequences from microbial amplicon reads. Nature Meth 10:996-998

Edgar RC, Haas BJ, Clemente JC, Quince C, Knight R (2011) UCHIME improves sensitivity and speed of chimera detection. Bioinformatics 27:2194-2200

Egan S, Harder T, Burke C, Steinberg P, Kjelleberg S, Thomas T (2013) The seaweed holobiont: understanding seaweed-bacteria interactions. FEMS Microbiol Rev 37:462-476

Hollants J, Leliaert F, De Clerck O, Willems A (2013) What we can learn from sushi: a review on seaweed-bacterial associations. FEMS Microbiol Ecol 83:1-16

Hossain MM, Tsuyumu S (2006) Flagella-mediated motility is required for biofilm formation by Erwinia carotovora subsp. carotovora. J Gen Plant Pathol 72:34-39

Houry A, Briandet R, Aymerich S, Gohar M (2010) Involvement of motility and flagella in Bacillus cereus biofilm formation. Microbiology 156:1009-1018

Laycock RA (1974) The detrital food chain based on seaweeds. I. Bacteria associated with the surface of Laminaria fronds. Mar Biol 25:223-234

Lemay MA, Martone PT, Keeling PJ, Burt JM, Krumhansl KA, Sanders RD, Wegener Parfrey L (2018) Sympatric kelp species share a large portion of their surface bacterial communities. Environ Microbiol 20:658-670

Louca S, Parfrey LW, Doebeli M (2016) Decoupling function and taxonomy in the global ocean microbiome. Science 353:1272-1277 
Mancuso FP, D’Hondt S, Willems A, Airoldi L, De Clerck O (2016) Diversity and temporal dynamics of the epiphytic bacterial communities associated with the canopy-forming seaweed Cystoseira compressa (Esper) Gerloff and Nizamuddin. Front Microbiol 7:476

Marzinelli EM, Campbell AH, Zozaya Valdes E, Vergés A, Nielsen S, Wernberg T, de Bettignies T, Bennett S, Caporaso JG, Thomas T, Steinberg PD (2015) Continental-scale variation in seaweed hostassociated bacterial communities is a function of host condition, not geography. Environ Microbiol 17:4078-4088

Mazure HG, Field JG (1980) Density and ecological importance of bacteria on kelp fronds in an upwelling region. J Exp Mar Biol Ecol 43: 173-182

Michelou VK, Caporaso JG, Knight R, Palumbi SR (2013) The ecology of microbial communities associated with Macrocystis pyrifera. PLoS One 8:e67480

Oksanen J, Blanchet F, Kindt R, Legendre P, O'hara R, Simpson G (2010) vegan: community ecology package. $R$ package version 1.17-4. 2010

Pruesse E, Quast C, Knittel K, Fuchs BM, Ludwig W, Peplies J, Glöckner FO (2007) SILVA: a comprehensive online resource for quality checked and aligned ribosomal RNA sequence data compatible with ARB. Nucleic Acids Res 35:7188-7196

Pujalte MJ, Lucena T, Ruvira MA, Arahal DR, Macián MC (2014) The family Rhodobacteraceae. In: Rosenberg E, DeLong EF, Lory S, Stackebrandt E, Thompson F (eds) The prokaryotes. Springer, Berlin, pp 439-512

Saha M, Weinberger F (2019) Microbial "gardening" by a seaweed holobiont: surface metabolites attract protective and deter pathogenic epibacterial settlement. J Ecol 107:2255-2265

Song J, Lim Y, Joung Y, Cho JC, Kogure K (2018) Rubritalea profundi sp. nov., isolated from deep-seawater and emended description of the genus Rubritalea in the phylum Verrucomicrobia. Int J Syst Evol Microbiol 68:1384-1389

Staufenberger T, Thiel V, Wiese J, Imhoff JF (2008) Phylogenetic analysis of bacteria associated with Laminaria saccharina. FEMS Microbiol Ecol 64:65-77

Tseng C (1994) Anthology of Tseng Cheng-Kui. Ocean Press, Beijing

Tsubouchi T, Shimane Y, Mori K, Miyazaki M, Tame A, Uematsu K, Maruyama T, Hatada Y (2013) Loktanella cinnabarina sp. nov., isolated from a deep subsea floor sediment, and emended description of the genus Loktanella. Int J Syst Evol Microbiol 63:13901395

Wang Q, Garrity GM, Tiedje JM, Cole JR (2007) Naive Bayesian classifier for rapid assignment of rRNA sequences into the new bacterial taxonomy. Appl Environ Microbiol 73:5261-5267
Wang G, Shuai L, Li Y, Lin W, Zhao X, Duan D (2008) Phylogenetic analysis of epiphytic marine bacteria on Hole-Rotten diseased sporophytes of Laminaria japonica. J Appl Phycol 20:403-409

Wang G, Lu B, Shuai L, Li D, Zhang R (2014) Microbial diseases of nursery and field-cultivated Saccharina japonica (Phaeophyta) in China. Algol Stud 145:39-51

Ward T,Larson J, Meulemans J, Hillmann B, Lynch J, Sidiropoulos D, Spear JR, Caporaso G, Blekhman R, Knight R, Fink R, Knights D et al. (2017) BugBase predicts organism level microbiome phenotypes. BioRxiv 133462

Weigel BL, Pfister CA (2019) Successional dynamics and seascape-level patterns of microbial communities on the canopy-forming kelps Nereocystis luetkeana and Macrocystis pyrifera. Front Microbiol 10:346

Wichham H (2016) ggplot2: Elegant Graphics for Data Analysis. Springer-Verlag, New York

Xiang JH (2001) Disease occurrence and control strategies of mariculture organisms. Ocean Press, Beijing (In Chinese)

Yoon J, Matsuo Y, Matsuda S, Adachi K, Kasai H, Yokota A (2007a) Rubritalea spongiae sp. nov. and Rubritalea tangerina sp. nov., two carotenoid- and squalene-producing marine bacteria of the family Verrucomicrobiaceae within the phylum 'Verrucomicrobia', isolated from marine animals. Int J Syst Evol Microbiol 57:2337-2343

Yoon J, Yasumoto-Hirose M, Matsuo Y, Nozawa M, Matsuda S, Kasai H, Yokota A (2007) Pelagicoccus mobilis gen. nov., sp. nov., Pelagicoccus albus sp. nov. and Pelagicoccus litoralis sp. nov., three novel members of subdivision 4 within the phylum 'Verrucomicrobia', isolated from seawater by in situ cultivation. Int J Syst Evol Microbiol 57:1377-1385

Yoon J, Matsuo Y, Adachi K, Nozawa M, Matsuda S, Kasai H, Yokota A (2008) Description of Persicirhabdus sediminis gen. nov., sp. nov., Roseibacillus ishigakijimensis gen. nov., sp. nov., Roseibacillus ponti sp. nov., Roseibacillus persicicus sp. nov., Luteolibacter pohnpeiensis gen. nov., sp. nov. and Luteolibacter algae sp. nov., six marine members of the phylum 'Verrucomicrobia', and emended descriptions of the class Verrucomicrobiae, the order Verrucomicrobiales and the family Verrucomicrobiaceae. Int $\mathrm{J}$ Syst Evol Microbiol 58:998-1007

Zhang R, Chang LR, Xiao LY, Zhang XY, Han Q, Li N, Egan S, Wang GG (2020) Diversity of the epiphytic bacterial communities associated with commercially cultivated healthy and diseased Saccharina japonica during the harvest season. J Appl Phycol 32:2071-2080

Publisher's note Springer Nature remains neutral with regard to jurisdictional claims in published maps and institutional affiliations. 